\title{
FADS2 Variants are Associated with Omega-6 Metabolic Conversion in Serbian Adults in a cross-sectional study - Implications for Precision Nutrition Practice
}

\author{
Manja Zec ( $\square$ manjazec@gmail.com ) \\ Institute for Medical Research, University of Belgrade https://orcid.org/0000-0001-5283-9295 \\ Ljiljana Stojković \\ Univerzitet u Beogradu Institut za nuklearne nauke Vinca \\ Milica Zeković \\ Institute for Medical Research, University of Belgrade \\ Biljana Pokimica \\ Institute for Medical Research, University of Belgrade \\ Maja Živković \\ Univerzitet u Beogradu Institut za nuklearne nauke Vinca \\ Aleksandra Stanković \\ Univerzitet u Beogradu Institut za nuklearne nauke Vinca \\ Maria Glibetic \\ Institute for Medical Research, University of Belgrade
}

\section{Research}

Keywords: arachidonic acid, desaturase-5, fatty acid desaturase genes, omega-6/omega-3 intake ratio, personalized nutrition, polyunsaturated fatty acids, single nucleotide polymorphisms.

Posted Date: December 18th, 2019

DOI: https://doi.org/10.21203/rs.2.19133/v1

License: (c) (i) This work is licensed under a Creative Commons Attribution 4.0 International License. Read Full License 


\section{Abstract}

Background High omega-6/omega-3 intake ratio in westernized world is of concern. FADS genes variants are associated with plasma long-chain polyunsaturated fatty acids (LC-PUFA) in diverse ethnicities and might modulate plasma omega-6/omega-3 net balance. Therefore, the objective of this study was to evaluate the relationships between FADS genetic variants with dietary fat and macronutrient intake, plasma phospholipid fatty acid profile, estimated plasma desaturase activity and cardiometabolic risk factors, in a sample of Serbian subjects.

Methods Non-smoking adult volunteers ( $>28$ years), free of acute or chronic disease were included. Food and nutrient data were compiled through $24 \mathrm{~h}$ recalls for non-consecutive days. Plasma phospholipid fatty acid content was assessed by gaschromatography. Selection of FADS2 variants (rs174593, rs174616 and rs174576) was based on its positional and functional aspect, and evidence-based data. Genotyping was performed by using Real-Time PCR. Estimated desaturase activities were calculated as conversion rates towards LC-PUFA in omega- 6 pathway. Multivariable-adjusted general linear were applied and the contribution of minor alleles to the variability of physiological parameters was analyzed by multivariable hierarchical multiple regression models.

Results Sample included 34 men and 54 women, mean age=40 \pm years, with $70 \%$ being overweight (BMl>25). Minor allele frequencies were $33 \%, 36 \%$ and $51 \%$ for rs 174593 , rs 174576 and rs 174616 , respectively, in line with other populations. None of the three variants was associated with food or nutrient intake, serum lipids, or obesity ( $p>0.05)$. Irrespective of gender, age, total daily polyunsaturated/saturated fatty acid intake and obesity, rs174593, rs174616 and rs174576 were associated with lower arachidonic acid (AA, C20:4 $n-6, p<0.001)$ and estimated desaturase-5 activity $(p<0.001)$ in plasma phospholipids. The rs 174576 associations with AA withstood multiple testing and additional adjustments for other variants.

Conclusion We observed inverse associations between FADS2 variants and plasma phospholipid AA but not omega-3 fatty acids in a sample of Serbian adults, and larger cohorts should confirm the associations. In our study FADS2 rs174576 exhibited the strongest associations, and future gene-dietary studies with varying omega-6/omega-3 intake should validate its suitability for precision nutrition strategies aimed at PUFA recommendations in Serbian population.Trial registration This is a cross-sectional study, forming part of larger intervention study registered at ClinicalTrials.gov as NCT02800967.

\section{Background}

Fatty acids are with prominent role in health and disease. Essential fatty acids include dietary linoleic acid (LA, C18:2 n-6) and alpha-linolenic fatty acid (aLNA, C18:3 n-3), and humans are incapable of endogenously synthesizing them. The LA and aLNA thus must be ingested through food and are further metabolically converted towards long-chain polyunsaturated fatty acids (LCPUFA). The LC-PUFA include arachidonic acid (AA, C20:4 n-6) with claimed proinflammatory properties [1], eicosapentaenoic acid (EPA, C20:5 n-3) with known anti-thrombotic, anti-arrhythmic, vasodilatory and other protective function [2], as well as docosahexaenoic acid (DHA, C22:6 n-3) with a crucial role in neuroplasticity and signal transduction [3]. The LC-PUFA function is associated with metabolic syndrome [4], cardiovascular disease [5], blood pressure [6] and allergies [7] across world-wide populations.

Essential fatty acids are metabolized to LC-PUFA by a set of metabolic steps, shared between omega- 6 ( $n-6)$ and omega-3 (n-3) pathway, and critical steps are catalyzed by desaturase- 5 and desaturase- 6 enzymes [8]. Desaturase- 6 is encoded by the FADS2 (fatty acid desaturase) gene and catalyzes the first downstream step from dietary LA and a-LNA to $y$-linolenic (C18:3 n-6) and stearidonic acid (C18:4 n-3), respectively [8, 9]. Desaturase-5 is encoded by the FADS1 gene and catalyzes rate-limiting conversion to AA and EPA from dihomo-y-linolenic (DGLA; C20:3 n-6) and C20:4 n-3, respectively [9]. The desaturase-6 is also utilized to produce DHA from EPA in a microsomal-peroxisomal pathway [9]. Tissue-specific LC-PUFA net balance thus depends on habitual diet, genetic function and gene-dietary interaction [10].

FADS1 and FADS2 variants (single nucleotide polymorphism - SNP) are associated with various health implications: reduced inflammation and cardiovascular disease risk [11], overweight and serum lipids, atopic disease, cognitive function and childmaternal health [12]. Minor allele presence in FADS gene cluster has repeatedly been linked with a decreased substrate-to-product ratio in fatty acid n-3 and n-6 pathways $[12,13]$. Pivotal genome-wide association studies reported associations between variants

Page 2/22 
in FADS1-2 genes and LC-PUFA in plasma and red blood cells $[14,15]$. The first FADS candidate gene study in 727 Caucasians identified associations between minor allele presence and LC-PUFA content in serum phospholipids [13]. Variants in FADS1 and FADS2 genes are associated with levels of LC-PUFA and desaturase activities in plasma and red blood cell phospholipids among 310 middle-aged healthy adults of mixed ethnicity [16]. Studies in Caucasian and East-Asian adults report FADS gene associations with estimated desaturation rates in serum $[17,18]$. The importance of investigating associations between common FADS variants and intermediary and clinical phenotypes in different populations in light of emerging precision nutrition practice, is summarized recently [10]. Taken together, variants in FADS genes modulate fatty acid levels and concomitant disease risk, conferring the role of the FADS variants as suitable nutrigenetic biomarkers.

Aside essential fatty acids, preformed LC-PUFA are ingested from food such as fish, nuts, eggs and meat. Ancestral dietary patterns presented with n-6 to n-3 intake ratio of less than 4:1. During the preceding decades, westernization of dietary patterns led to dramatic increase in the ratio, resulting in $15: 1$ and higher $n-6 / n-3$ composition of habitual intake in contemporary populations, mostly attributed to cooking practices based on immense utilization of n-6 LA-rich vegetable oils, such as corn, soybean and canola oil [19]. In addition, westernized diet is low in n-3 rich fatty fish. Disturbed dietary n-6/n-3 ratio leads to metabolic shift towards long-chain n-6 products in place of long-chain n-3 products, which affects cardiometabolic risk such as obesity [20]. Lower levels of LC-PUFA metabolic products are associated with detrimental effects with regards to brain [3], immune function and inflammation [21]. Variants in FADS genes might alleviate health risk of the high $n-6 / n-3$ ratio, by shifting endogenous conversion towards a more favorable balance. Precision nutrition concept suggests tailoring of recommendations for PUFA supply in subjects or groups with specific FADS hallmarks [11, 12].

Ethnic-specific FADS1 and FADS2 gene effects have been demonstrated [22-24], but no study investigated FADS variants and its effects on fatty acid metabolism and cardio-metabolic risk factors in Serbian population. Therefore, the purpose of this study was to evaluate the FADS2 genetic variants: rs174593, rs174616 and rs174576 as candidates for nutrigenetic-based precision nutrition practice, by investigating their relationship with dietary fat and macronutrient intake, plasma phospholipid fatty acid profile, estimated plasma desaturase activity and cardiometabolic risk factors (serum lipids and obesity), in a sample of Serbian adults. Based on literature data, we hypothesize that the minor allele within the variants will be associated with lower product-to-precursor ratio in fatty acid metabolic cascades.

\section{Subjects And Methods}

\section{Ethical Clearance and Study Population}

Current study formed part of a larger interventional study registered at ClinicalTrials.gov as NCT02800967. The study protocol adhered to the regulations of the 1975 Declaration of Helsinki and is approved by the Clinical Hospital Center in Zemun (Belgrade, Serbia), Ethics Committee Approval, No: 2125, 2013. Study subjects were recruited by means of newspaper advertisement, word of mouth or at the clinic. Interested volunteers who were non-smoking adults (>28 years) with no signs of clinical disease were considered eligible. Exclusion criteria were presence of any acute or chronic illness, smoking, lactation and pregnancy, allergies, regularly prescribed medication use related to cardiovascular risk factors (including statins) and unwillingness to participate in a genetic study. None of the herein variants studied succumbs necessary reporting due to incidental findings [25]. Following an informed interview with the study team, eligible volunteers were asked to sign the informed consent form, approved together with the study protocol. Informed participation included willingness to participate in a candidate-gene study.

\section{Assessment of dietary and clinical data}

Dietary intake was assessed with repeated $24 \mathrm{~h}$ recalls for two non-consecutive days to allow correction for within-subject food consumption variability. In-depth face-to-face interviews were conducted by trained researchers according to a standardized multiple-pass methodological approach. Dietary assessment was complemented with a variety of self-reported sociodemographic, lifestyle and other related data and performed in the study examination site. Questionnaires were analyzed by Diet Assess \& Plan nutritional platform for standardized food consumption data collection and diet evaluation [26]. We calculated total daily caloric and nutrient intake using Serbian Food Composition Database, compliant with EuroFIR standards [27]. Subjects consumed no n-3 dietary supplementation in any nutraceutical form. Thus, reported n-3 intake is due to the food intake solely. Among food groups, we analyzed fish and nuts as recognized n-3 dietary sources.

Page 3/22 
Anthropometry parameters were measured in a private room, and subjects were asked to stay in light clothing. Height and weight were measured with a precision to the nearest $0.1 \mathrm{~cm}$ and $0.1 \mathrm{~kg}$, respectively. Height was measured by a portable stadiometer, and weight was assessed by bioimpedance analyzer TANITA UM072 balance (TANITA Health Equipment H.K. LTD). Waist circumference was evaluated by flexible measurer. The BMI was calculated as weight $(\mathrm{kg}) /(\mathrm{height}[\mathrm{m}])^{2}$.

\section{Blood samples collection and analysis}

After an overnight fast of 10 hours refraining from food and beverage, blood samples were collected in tubes with ethylenediaminetetraacetic acid as an anticoagulant. Blood collection was performed between 7-8 a.m. by venepuncture of the antecubital vein. Serum lipid parameters and glucose concentration were determined on the same day when collected by use of Roche Diagnostics' kits and clinical chemistry analyzer Cobas c111 (Roche Diagnostics, Basel, Switzerland), according to the manufacturer's instructions.

Fatty acid concentrations were determined by gas chromatography, as previously described in [28] with slight modifications. Briefly, total plasma lipids were extracted according to the method of Folch [29] by chloroform-methanol mixture (2:1). Among total lipid pool, phospholipids were further separated on thin-layer chromatography silica plate using the system of petroleum ether, diethyl ether and glacial acetic acid (87:12:1, by volume). The phospholipid fraction was scraped into glass tubes and fatty acid methyl-esters obtained by direct trans-methylation. Resulting fatty acid methyl esters were analyzed by gas chromatography on Shimadzu chromatograph GC 2014, Tokyo, Japan equipped with a flame ionization detector and a Rtx 2330 fused silica gel capillary column (60 m $\times 0.25 \mathrm{~mm}$ id $\times 0.2 \mu \mathrm{m}$ film thickness) (Restek Co., Bellefonte, PA, USA). The adequate separation was obtained with an initial temperature of the column of $140{ }^{\circ} \mathrm{C}$ for $5 \mathrm{~min}$, then increased to $220^{\circ} \mathrm{C}$, at $3^{\circ} \mathrm{C} / \mathrm{min}$ rate, and final temperature held for $20 \mathrm{~min}$. We compared the peak retention times to certified calibration mixtures after 51 min (PUFA-2, Supelco, Bellefonte, PA, USA, and 37 FAMEs mix, Sigma Chemical Co., St. Louis, MO, USA), and areas under curves were calculated. For the purposes of current study we identified following fatty acids as percentage of total phospholipid pool in plasma: palmitic acid (C16:0), palmitoleic acid (C16:1 n-7), staeric acid (C18:0), vaccenic acid (C18:1 n-7), oleic acid (18:1n-9), LA, DGLA, AA, adrenic acid (C22:4n-6), EPA, docosapentaenoic acid (C22:5n-3), and DHA. Estimated desaturase activities were calculated based on the conversion rates towards long-chain unsaturated products in $n-6$ pathway [30]. Thus, we predicted desaturase-5 activity by use of AA/DGLA ratio in plasma phospholipids. For estimation of desaturase- 6 activity we used an aggregate enzymatic index of DGLA/LA to include additional elongation step.

\section{SNP selection and genotyping}

During SNP selection we accounted for its positional and functional aspect, together with evidence-based data on SNP relevance. We finally selected SNPs located in different introns of FADS2 gene: rs174576 (intron 1), rs174593 (intron 5) and rs174616 (intron 7), after analysis of data from the above-mentioned studies and taking into account all following criteria: 1) consistent effect of each SNP on LC-PUFA plasma/serum levels across the analyzed studies; 2) significant association (with correction for multiple testing where appropriate) between each stated SNP and LC-PUFA plasma/serum levels after adjustment for age, gender, ethnicity, BMI, dietary LC-PUFA and energy intake from fat 3) from a pair of the quoted SNPs if linkage disequilibrium (LD) was found to be high $\left(r^{2} \geq 0.8\right)$ (pairwise LD between SNPs was calculated using SNP Annotation and Proxy Search (SNAP) database: https://omictools.com/snp-annotation-and-proxy-search-tool, [31]), we selected one SNP for which we revealed a high RegulomeDB score (score categories 1 and 2, out of 6), predicting a SNP position within transcriptional regulatory DNA element (http://www.regulomedb.org, [32]). Selected SNPs were each with a minor allele frequency of above $10 \%$ within European populations, according to SNP database of National Center for Biotechnology Information (http://www.ncbi.nlm.nih.gov/snp/) and comparable to the data from 1000 Genome Study project.

Genomic DNA was isolated from whole peripheral blood samples collected with ethylenediaminetetraacetic acid, by a method based on phenol-chloroform extraction [33]. TaqMan ${ }^{\circledR}$ SNP genotyping assays (Applied Biosystems Inc, Foster City, CA, USA) were used for detection of the selected FADS2 intronic variants: C__2575520_10 (rs174576), C__2575513_10 (rs174593) and C__2268923_10 (rs174616), according to the manufacturer's instructions. The 7500 Real-Time PCR System with SDS v1.4.0 software (Applied Biosystems Inc, Foster City, CA, USA) was employed for the performance of PCR and determination of genotypes.

\section{Statistical Analysis}

Page $4 / 22$ 
Statistical analysis was performed by SPSS ver. 24 (Chicago II), and probability threshold of 0.05 was relevant. Normally distributed data are presented as mean $\pm S D$, otherwise median (interquartile range). Distribution of genotypes was tested using Chi-square test with $1 \mathrm{df}$, to evaluate deviations from Hardy-Weinberg equilibrium, with probability threshold set at 0.05 . Two subjects were with missing dietary data. Missing data were completely at random and were included in the analyses by intentionto-treat principle, accounted for in a statistical syntax. For each SNP, dominant model was applied to group subjects according to presence of at least one minor allele at a single group and compare with null allele homozygotes. We then applied general linear models, adjusted for age, gender, energy intake and obesity, to evaluate whether there are different distributions of parameters between major allele homozygotes and minor allele carriers. The outcome variables were structured based on their physiological and clinical relevance on parameters of food and nutrient intake, fatty acid profile in plasma phospholipids, fatty acid conversion indices and cardio-metabolic parameters, and the results are presented accordingly. If a biomarker exhibited significantly different distribution in the dominant genetic model, we applied additive genetic model to test associations with intermediary phenotype and cardiometabolic risk factors with addition of a minor allele per variant. In order to evaluate the contribution of minor alleles to the variability of selected physiological parameters we applied hierarchical multiple regression models, fully adjusted for gender, age and total daily polyunsaturated/saturated fatty acid intake and obesity. We visually inspected the distribution of residuals to satisfy the rule of linearity, and log-transformed variables if necessary. If upon multivariable regression analyses variants were significantly associated with an outcome, we additionally adjusted for the remaining two variants to check for the independent gene effects. To account for multiple testing in regression analysis, we applied conservative Bonferroni correction for 3 exposure variants, hence $p$-value of less than 0.017 denoted level of statistical significance.

\section{Results}

\section{Study subjects' characteristics}

The study sample included 54 women and 34 men, mean age of $40.49 \pm 7.03$ years (Table 1 ). In the complete sample, there were $70.5 \%$ overweight subjects. Subjects were with balanced macronutrient intake, with median daily energy intake of $44.24 \%, 15.95 \%$, $40.18 \%$ from carbohydrates, proteins and fats, respectively. Estimated median percentage contributions of monounsaturated, polyunsaturated and saturated fatty acids to daily energy intake were $12.55 \%, 10.07 \%$, and $12.82 \%$, respectively. 
Table 1

Characteristics of study subjects across tertiles (median [interquartile range]) of omega-6/omega-3 habitual intake

\begin{tabular}{|c|c|c|c|c|c|}
\hline & $\begin{array}{l}\text { Complete Sample, } n \\
88 \\
16.29[12.31,24.56]\end{array}$ & $\begin{array}{l}\text { Tertile 1, n } 28 \\
11.04[7.90,12.31]\end{array}$ & $\begin{array}{l}\text { Tertile 2, n } 29 \\
15.93[14.02,18.63]\end{array}$ & $\begin{array}{l}\text { Tertile 3, n } 29 \\
26.62[24.09,35.49]\end{array}$ & $\begin{array}{l}\mathrm{p} \text { - } \\
\text { value }\end{array}$ \\
\hline Women, n (\%) & $54(61.4)$ & $16(57.1)$ & $19(65.5)$ & $18(62.1)$ & 0.810 \\
\hline Age, years & $40.49 \pm 7.03$ & $41.05 \pm 8.03$ & $41.45 \pm 6.99$ & $39.72 \pm 5.69$ & 0.616 \\
\hline Overweight, n (\%) & $62(70.5)$ & $17(60.7)$ & $22(75.9)$ & $21(72.4)$ & 0.433 \\
\hline $\begin{array}{l}\text { Energy intake, } \\
\mathrm{kcal} / \text { day }\end{array}$ & $\begin{array}{l}\text { 1916.13 [1596.20, } \\
2408.33]\end{array}$ & $\begin{array}{l}\text { 1875.45 [1521.93, } \\
2387.69]\end{array}$ & $\begin{array}{l}\text { 1911.91 [1607.02, } \\
\text { 2254.82] }\end{array}$ & $\begin{array}{l}2066.71[1588.13 \\
2589.06]\end{array}$ & 0.576 \\
\hline \multicolumn{6}{|c|}{ Dietary and nutrient intake, $\mathrm{g} /$ day } \\
\hline Carbohydrates & $\begin{array}{l}212.95[176.47, \\
259.98]\end{array}$ & $\begin{array}{l}187.90[164.91, \\
245.15]\end{array}$ & $\begin{array}{l}197.49[176.81, \\
257.60]\end{array}$ & $\begin{array}{l}227.19[199.78, \\
285.12]\end{array}$ & 0.101 \\
\hline Protein & $78.88[62.74,96.90]$ & $78.80[62.04,99.51]$ & $79.89[65.72,94.48]$ & $78.69[60.32,95.03]$ & 0.945 \\
\hline Fat & $\begin{array}{l}84.70[65.73 \\
113.11]\end{array}$ & $\begin{array}{l}87.44[68.54 \\
114.23]\end{array}$ & $\begin{array}{l}84.17[65.60 \\
108.46]\end{array}$ & $\begin{array}{l}82.71[60.35 \\
115.00]\end{array}$ & 0.927 \\
\hline MUFA & $25.37[21.42,36.06]$ & $26.48[21.59,35.73]$ & $24.31[21.09,35.20]$ & $26.17[19.47,37.66]$ & 0.885 \\
\hline PUFA & $22.24[15.36,30.48]$ & $26.21[18.31,31.15]$ & $19.94[15.06,30.54]$ & $21.74[13.93,26.52]$ & 0.502 \\
\hline SFA & $27.00[21.10,37.26]$ & $27.22[19.21,34.94]$ & $26.19[21.93,37.12]$ & $27.56[20.69,39.19]$ & 0.692 \\
\hline PUFA/SFA & $0.72[0.60,1.05]$ & $0.93[0.61,1.29]$ & $0.68[0.57,1.05]$ & $0.70[0.61,0.91]$ & 0.200 \\
\hline Lauric acid, 12:0 & $0.94[0.55,1.26]$ & $0.72[0.36,1.21]$ & $0.91[0.66,1.23]$ & $1.08[0.65,1.69]$ & 0.158 \\
\hline Myristic acid, 14:0 & $2.16[1.53,3.26]$ & $1.71[1.20,3.25]$ & $2.22[1.80,2.87]$ & $2.35[1.52,3.64]$ & 0.600 \\
\hline Palmitic acid, 16:0 & $13.51[10.68,18.63]$ & $13.52[10.04,18.02]$ & $13.47[11.34,18.64]$ & $13.54[10.89,19.93]$ & 0.770 \\
\hline Stearic acid, 18:0 & $6.52[4.61,8.70]$ & $6.50[4.39,7.92]$ & $6.06[4.42,8.56]$ & $7.57[5.38,10.35]$ & 0.230 \\
\hline Oleic acid, 18:1 n-9 & $21.03[17.00,29.12]$ & $20.81[17.27,28.94]$ & $20.32[16.61,28.89]$ & $22.59[16.12,32.42]$ & 0.873 \\
\hline $\begin{array}{l}\text { Linoleic acid, 18:2 } \\
n-6\end{array}$ & $17.81[13.97,23.04]$ & $18.44[15.03,22.05]$ & $16.49[13.95,21.58]$ & $19.11[13.04,26.77]$ & 0.329 \\
\hline $\begin{array}{l}\text { a-linolenic acid, } \\
18: 3 n-3\end{array}$ & $0.74[0.50,1.20]$ & $1.21[0.75,1.48]$ & $0.73[0.56,1.15]$ & $0.50[0.40,0.76]$ & $\varangle 0.001$ \\
\hline DGLA, 20:3 n-6 & $0.01[0.00,0.01]$ & $0.00[0.00,0.01]$ & $0.01[0.01,0.02]$ & $0.01[0.01,0.01]$ & 0.108 \\
\hline $\begin{array}{l}\text { Arachidonic acid, } \\
20: 4 \text { n-6 }\end{array}$ & $0.13[0.09,0.21]$ & $0.11[0.08,0.17]$ & $0.15[0.10,0.24]$ & $0.18[0.10,0.21]$ & 0.135 \\
\hline EPA, $20: 5 n-3$ & $0.03[0.01,0.14]$ & $0.23[0.06,0.57]$ & $0.02[0.01,0.07]$ & $0.01[0.00,0.06]$ & $\varangle 0.001$ \\
\hline DHA, 22:6 n-3 & $0.17[0.09,0.43]$ & $0.45[0.17,0.81]$ & $0.14[0.10,0.34]$ & $0.09[0.04,0.17]$ & $\varangle 0.001$ \\
\hline Omega-6 & $17.94[14.16,23.24]$ & $18.52[15.11,22.21]$ & $16.62[14.17,21.69]$ & $19.21[13.17,26.99]$ & 0.334 \\
\hline
\end{tabular}

Data are presented as mean \pm SD or median [interquartile range], depending on the distribution, and categorical variables are presented as percentage of whole. P values denote probability trends upon general linear model and Kruskal-Wallis analyses, for parametric and non-parametric approach, respectively; testing difference between parameter distributions across tertiles of daily omega-6 to omega-3 intake ratio [g]. Bolded text denotes statistical significance.

DGLA, dihomo-y-linolenic acid; EPA, eicosapentaenoic acid; DPA, docosapentaenoic acid; DHA, docosahexaenoic acid; Omega6, Total Omega-6 intake; Omega-3, Total Omega-3 intake; Marine omega-3, Cumulative intake of EPA and DHA; MUFA, Intake of monounsaturated fatty acids; PUFA, Intake of polyunsaturated fatty acids; SFA, Intake of saturated fatty acids; HDL-c, High density lipoprotein cholesterol; LDL-c, Low density lipoprotein cholesterol; TAG, triglycerides. 


\begin{tabular}{|c|c|c|c|c|c|}
\hline & $\begin{array}{l}\text { Complete Sample, } n \\
88 \\
16.29[12.31,24.56]\end{array}$ & $\begin{array}{l}\text { Tertile 1, n } 28 \\
11.04[7.90,12.31]\end{array}$ & $\begin{array}{l}\text { Tertile 2, n } 29 \\
15.93[14.02,18.63]\end{array}$ & $\begin{array}{l}\text { Tertile 3, n } 29 \\
26.62[24.09,35.49]\end{array}$ & $\begin{array}{l}\mathrm{p}- \\
\text { value }\end{array}$ \\
\hline Omega-3 & $1.06[0.75,1.69]$ & $1.95[1.33,2.76]$ & $1.07[0.84,1.37]$ & $0.68[0.48,0.91]$ & $\llbracket 0.001$ \\
\hline Marine omega-3 & $0.19[0.10,0.65]$ & $0.72[0.19,1.27]$ & $0.18[0.11,0.42]$ & $0.11[0.07,0.21]$ & $\varangle 0.001$ \\
\hline Fish & $28.34[0.00,70.30]$ & $\begin{array}{l}66.67[11.75 \\
141.66]\end{array}$ & $26.67[0.00,74.17]$ & $0.00[0.00,46.67]$ & $\nabla 0.001$ \\
\hline Nuts & $9.26[2.15,20.83]$ & $15.59[2.57,28.20]$ & $12.68[4.30,20.11]$ & $7.10[2.15,13.21]$ & 0.358 \\
\hline \multicolumn{6}{|c|}{ Fatty acids in plasma phospholipids, $\%$ of total pool } \\
\hline Palmitic acid, 16:0 & $30.32 \pm 2.10$ & $30.24 \pm 1.80$ & $30.65 \pm 2.65$ & $30.11 \pm 1.78$ & 0.622 \\
\hline $\begin{array}{l}\text { Palmitoleic acid, } \\
\text { 16:1 n-7 }\end{array}$ & $0.54[0.42,0.72]$ & $0.51[0.41,0.66]$ & $0.61[0.45,0.79]$ & $0.61[0.42,0.77]$ & 0.576 \\
\hline Stearic acid, 18:0 & $16.60 \pm 1.49$ & $16.54 \pm 1.36$ & $16.69 \pm 1.73$ & $16.47 \pm 1.36$ & 0.860 \\
\hline Oleic acid, 18:1 n-9 & $7.76[7.24,8.69]$ & $7.67[7.26,8.21]$ & $8.19[7.26,9.06]$ & $7.81[7.23,8.82]$ & 0.380 \\
\hline $\begin{array}{l}\text { Vaccenic acid, 18:1 } \\
\text { n-7 }\end{array}$ & $2.43[2.10,2.78]$ & $2.44[2.12,2.78]$ & $2.45[2.06,2.82]$ & $2.39[2.13,2.71]$ & 0.993 \\
\hline $\begin{array}{l}\text { Linoleic acid, 18:2 } \\
\mathrm{n}-6\end{array}$ & $23.99 \pm 3.24$ & $24.02 \pm 3.13$ & $23.29 \pm 3.12$ & $24.63 \pm 3.56$ & 0.328 \\
\hline DGLA, $20: 3$ n-6 & $2.97 \pm 0.91$ & $2.66 \pm 0.83$ & $3.29 \pm 0.80$ & $3.02 \pm 1.01$ & 0.033 \\
\hline $\begin{array}{l}\text { Arachidonic acid, } \\
20: 4 \text { n-6 }\end{array}$ & $10.91 \pm 2.11$ & $11.28 \pm 1.69$ & $10.61 \pm 2.14$ & $10.69 \pm 2.40$ & 0.432 \\
\hline EPA, 20:5 n3 & $0.30[0.21,0.43]$ & $0.37[0.26,0.43]$ & $0.30[0.22,0.49]$ & $0.26[0.18,0.38]$ & 0.177 \\
\hline $\begin{array}{l}\text { Adrenic acid, 22:4 } \\
\mathrm{n}-6\end{array}$ & $0.42[0.35,0.51]$ & $0.40[0.34,0.48]$ & $0.43[0.33,0.56]$ & $0.42[0.35,0.56]$ & 0.279 \\
\hline DPA, 22:5 n-3 & $0.56[0.46,0.66]$ & $0.61[0.48,0.77]$ & $0.53[0.48,0.65]$ & $0.55[0.44,0.62]$ & 0.201 \\
\hline DHA, 22:6 n-3 & $2.85 \pm 0.88$ & $3.09 \pm 0.90$ & $2.90 \pm 1.01$ & $2.57 \pm 0.65$ & 0.091 \\
\hline Desaturase-6 & $0.12[0.09,0.15]$ & $0.10[0.08,0.15]$ & $0.14[0.12,0.17]$ & $0.12[0.09,0.14]$ & 0.070 \\
\hline Desaturase-5 & $3.71[2.97,4.88]$ & $4.35[3.25,5.93]$ & $3.06[2.71,3.93]$ & $3.67[2.77,4.88]$ & 0.023 \\
\hline AA/EPA & $34.53[26.23,51.73]$ & $31.09[25.05,42.87]$ & $33.40[16.74,52.74]$ & $39.74[28.64,57.43]$ & 0.240 \\
\hline AADHA & $3.91[3.29,4.93]$ & $3.86[3.04,4.70]$ & $3.51[3.12,4.84]$ & $3.99[3.47,4.95]$ & 0.531 \\
\hline \multicolumn{6}{|l|}{ Clinical parameters } \\
\hline Glucose, mmol/L & $4.87[4.59,5.31]$ & $4.77[4.49,5.28]$ & $4.94[4.58,5.40]$ & $4.92[4.68,5.19]$ & 0.554 \\
\hline TAG, mmol/L & $1.12[0.75,1.52]$ & $1.18[0.77,1.43]$ & $1.28[0.76,1.86]$ & $0.98[0.67,1.44]$ & 0.111 \\
\hline $\begin{array}{l}\text { Total Cholesterol, } \\
\mathrm{mmol} / \mathrm{L}\end{array}$ & $5.24 \pm 1.01$ & $5.58 \pm 1.08$ & $5.52 \pm 0.97$ & $4.70 \pm 0.73$ & 0.001 \\
\hline
\end{tabular}

Data are presented as mean \pm SD or median [interquartile range], depending on the distribution, and categorical variables are presented as percentage of whole. $P$ values denote probability trends upon general linear model and Kruskal-Wallis analyses, for parametric and non-parametric approach, respectively; testing difference between parameter distributions across tertiles of daily omega-6 to omega-3 intake ratio [g]. Bolded text denotes statistical significance.

DGLA, dihomo-y-linolenic acid; EPA, eicosapentaenoic acid; DPA, docosapentaenoic acid; DHA, docosahexaenoic acid; Omega6, Total Omega-6 intake; Omega-3, Total Omega-3 intake; Marine omega-3, Cumulative intake of EPA and DHA; MUFA, Intake of monounsaturated fatty acids; PUFA, Intake of polyunsaturated fatty acids; SFA, Intake of saturated fatty acids; HDL-c, High density lipoprotein cholesterol; LDL-c, Low density lipoprotein cholesterol; TAG, triglycerides. 


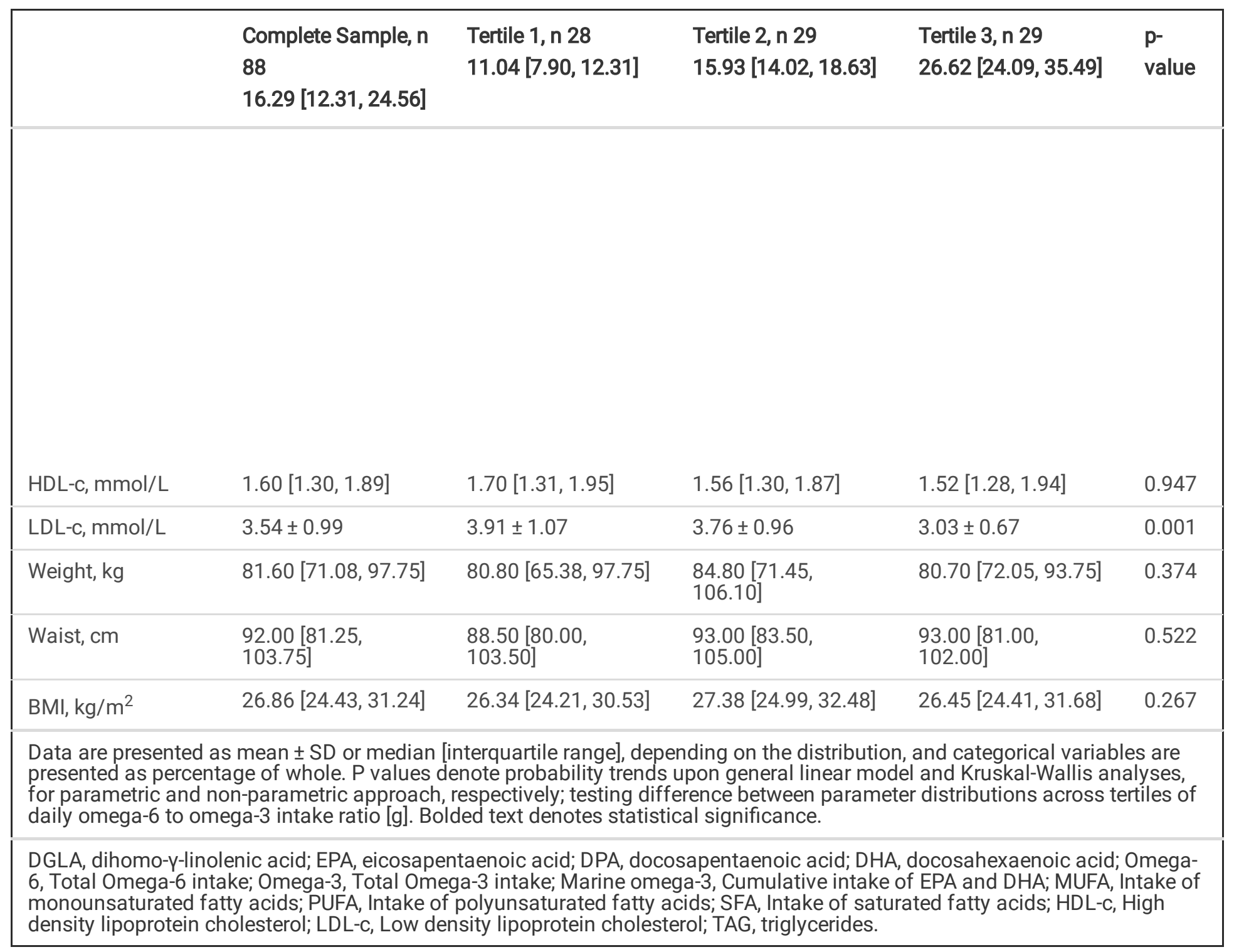

In the total sample, median n-6/n-3 intake ratio was 16.29 (Table 1). Daily consumption of macronutrients and fat groups was equally distributed across tertiles of n-6/n-3 intake. Intake of individual n-3 fatty acids, aLNA, EPA and DHA, decreased with increasing tertiles of the ratio $(p<0.001)$, while no differences in distribution were seen among dietary $n-6$ fatty acid. Intake of fish decreased across the tertiles, with as much as $69 \%$ of the subjects in the highest tertile eating no fish at all. Among fatty acids in plasma phospholipids only DGLA increased with increasing tertiles of $n-6 / n-3$. Other plasma fatty acids were equally distributed across the tertiles of n-6/n-3 consumption, although DHA tended to decrease without reaching significance $(p=0.091)$. The predicted activity of desaturase -5 decreased with increasing tertiles of the ratio $(p=0.023)$ (Table 1$)$. Increased $n-6 / n-3$ intake ratio was followed by lower total and LDL-c cholesterol levels $(p=0.001$ for both, Table 1$)$.

For the three selected FADS2 SNPs, distribution of minor allele carriers was equal across the tertiles of the $n-6 / n-3$ intake ratio (Supplementary Table 1).

\section{FADS2 allele and genotype distribution}

Genotype frequencies of the three selected FADS2 variants conformed to Hardy-Weinberg balance (Table 2). Allele and genotype frequencies are presented in Table 2. Minor allele frequencies were $0.33,0.36$ and 0.51 for rs 174593 , rs 174576 and rs 174616 , respectively. 
Table 2

Allele and genotype distribution of the FADS2 genetic variants in 88 Serbian adults

\begin{tabular}{|c|c|c|c|c|c|c|c|c|}
\hline & $\begin{array}{l}\text { Chromosome } \\
\text { Position }\end{array}$ & $\begin{array}{l}\text { Major allele } \\
\text { homozygote } \\
\text { n (\%) }\end{array}$ & $\begin{array}{l}\text { Heterozygote } \\
\text { n (\%) }\end{array}$ & $\begin{array}{l}\text { Minor allele } \\
\text { homozygote } \\
\text { n (\%) }\end{array}$ & $\begin{array}{l}\text { Minor } \\
\text { allele } \\
\text { carriers } \\
\mathrm{n}(\%)\end{array}$ & $\begin{array}{l}\text { Minor Allele } \\
\text { Frequency } \\
\text { Our } \\
\text { Study/1000Genome }\end{array}$ & $\begin{array}{l}\text { Chi- } \\
\text { sq }\end{array}$ & $\begin{array}{l}\mathrm{p} \text { - } \\
\text { value }\end{array}$ \\
\hline $\begin{array}{l}\text { rs } 174593 \\
T>C\end{array}$ & 11:61851359 & $\begin{array}{l}\text { TT } \\
37(42)\end{array}$ & $\begin{array}{l}\mathrm{TC} \\
44(50)\end{array}$ & $\begin{array}{l}\mathrm{CC} \\
7(8)\end{array}$ & $\begin{array}{l}\mathrm{TC}+ \\
\mathrm{CC} \\
51(58)\end{array}$ & $0.33 / 0.21$ & 1.71 & 0.191 \\
\hline $\begin{array}{l}\text { rs } 174616 \\
G>A\end{array}$ & $11: 61861650$ & $\begin{array}{l}\text { GG } \\
18(20)\end{array}$ & $\begin{array}{l}\text { GA } \\
50(57)\end{array}$ & $\begin{array}{l}\text { AA } \\
20(23)\end{array}$ & $\begin{array}{l}\text { GA + } \\
\text { AA } \\
70 \\
(79.5)\end{array}$ & $0.51 / 0.48$ & 1.99 & 0.158 \\
\hline $\begin{array}{l}\text { rs174576, } \\
C>A\end{array}$ & 11:61836038 & $\begin{array}{l}\mathrm{CC} \\
33(37.5)\end{array}$ & $\begin{array}{l}\text { CA } \\
46(52)\end{array}$ & $\begin{array}{l}\text { AA } \\
9(10)\end{array}$ & $\begin{array}{l}\text { CA+ } \\
\text { AA } \\
55 \\
(62.5)\end{array}$ & $0.36 / 0.36$ & 1.72 & 0.189 \\
\hline
\end{tabular}

\section{Distribution of food and nutrient intake with regards to FADS2 genotypes}

There were no differences in daily consumption of any macronutrient, fat group, individual fatty acids, fish or nut intake, dependent on FADS2 minor alleles' presence (Supplementary Table 2).

Distribution of fatty acids and conversion factors in plasma phospholipids with regards to FADS2 genotypes

Subjects carrying minor rs174593 C allele presented with lower palmitoleic $(p=0.046)$ and higher stearic acid $(p=0.034)$ in comparison with major allele homozygotes (Table 3). Subjects carrying minor rs174576 A allele tended to be with lower palmitoleic acid $(p=0.056)$. 
Table 3

Distribution of fatty acids in plasma phospholipids according to FADS2 genotypes in Serbian adults.

\begin{tabular}{|c|c|c|c|c|c|c|c|c|c|}
\hline & rs174593 & & & rs174616 & & & rs174576 & & \\
\hline & $\begin{array}{l}\text { TT } \\
\text { n } 37\end{array}$ & $\begin{array}{l}\mathrm{TC}+\mathrm{CC} \\
\mathrm{n} 51\end{array}$ & $\begin{array}{l}\mathrm{p}- \\
\text { value }\end{array}$ & $\begin{array}{l}\text { GG } \\
\text { n } 18\end{array}$ & $\begin{array}{l}\mathrm{GA}+\mathrm{AA} \\
\mathrm{n} 70\end{array}$ & $\begin{array}{l}p- \\
\text { value }\end{array}$ & $\begin{array}{l}\mathrm{CC} \\
\text { n } 33\end{array}$ & $\begin{array}{l}\mathrm{CA}+\mathrm{AA} \\
\mathrm{n} 55\end{array}$ & $\begin{array}{l}\mathrm{p}- \\
\text { value }\end{array}$ \\
\hline $\begin{array}{l}\text { Palmitic acid, } \\
16: 0\end{array}$ & $\begin{array}{l}30.3 \pm \\
1.80\end{array}$ & $\begin{array}{l}30.31 \pm \\
2.31\end{array}$ & 0.950 & $\begin{array}{l}30.41 \pm \\
2.35\end{array}$ & $\begin{array}{l}30.30 \pm \\
2.06\end{array}$ & 0.788 & $\begin{array}{l}30.29 \pm \\
1.88\end{array}$ & $\begin{array}{l}30.34 \pm \\
2.24\end{array}$ & 0.843 \\
\hline $\begin{array}{l}\text { Palmitoleic acid, } \\
16: 1 \text { n-7 }\end{array}$ & $\begin{array}{l}0.64 \\
{[0.48} \\
0.78]\end{array}$ & $\begin{array}{l}0.50 \\
{[0.41} \\
0.67]\end{array}$ & 0.046 & $\begin{array}{l}0.61 \\
{[0.53} \\
0.68]\end{array}$ & $\begin{array}{l}0.53 \\
{[0.41} \\
0.75]\end{array}$ & 0.987 & $\begin{array}{l}0.64 \\
{[0.50} \\
0.77]\end{array}$ & $\begin{array}{l}0.50 \\
{[0.40} \\
0.67]\end{array}$ & 0.056 \\
\hline Stearic acid, 18:0 & $\begin{array}{l}16.32 \pm \\
1.65\end{array}$ & $\begin{array}{l}16.80 \pm \\
1.33\end{array}$ & 0.034 & $\begin{array}{l}17.03 \pm \\
1.65\end{array}$ & $\begin{array}{l}16.51 \pm \\
1.44\end{array}$ & 0.246 & $\begin{array}{l}16.84 \pm \\
1.66\end{array}$ & $\begin{array}{l}16.45 \pm \\
1.36\end{array}$ & 0.693 \\
\hline $\begin{array}{l}\text { Oleic acid, 18:1 } \\
\text { n-9 }\end{array}$ & $\begin{array}{l}7.69 \\
{[7.09} \\
8.36]\end{array}$ & $\begin{array}{l}7.81 \\
{[7.31} \\
8.79]\end{array}$ & 0.342 & $\begin{array}{l}7.63 \\
{[7.32} \\
8.36]\end{array}$ & $\begin{array}{l}7.79 \\
{[7.08} \\
8.73]\end{array}$ & 0.569 & $\begin{array}{l}7.65 \\
{[7.24} \\
8.32]\end{array}$ & $\begin{array}{l}7.77 \\
{[7.23} \\
8.81]\end{array}$ & 0.933 \\
\hline $\begin{array}{l}\text { Vaccenic acid, } \\
18: 1 \mathrm{n}-7\end{array}$ & $\begin{array}{l}2.44 \\
{[2.17} \\
2.68]\end{array}$ & $\begin{array}{l}2.37 \\
{[2.04} \\
2.83]\end{array}$ & 0.738 & $\begin{array}{l}2.63 \\
{[2.13} \\
2.83]\end{array}$ & $\begin{array}{l}2.36 \\
{[2.08} \\
2.78]\end{array}$ & 0.691 & $\begin{array}{l}2.44 \\
{[2.12} \\
2.67]\end{array}$ & $\begin{array}{l}2.38 \\
{[2.02} \\
2.85]\end{array}$ & 0.570 \\
\hline $\begin{array}{l}\text { Linoleic acid, } \\
\text { 18:2 n-6 }\end{array}$ & $\begin{array}{l}23.24 \pm \\
3.13\end{array}$ & $\begin{array}{l}24.53 \pm \\
3.24\end{array}$ & 0.184 & $\begin{array}{l}22.44 \pm \\
2.64\end{array}$ & $\begin{array}{l}24.33 \pm \\
3.28\end{array}$ & 0.063 & $\begin{array}{l}22.65 \pm \\
3.00\end{array}$ & $\begin{array}{l}24.82 \pm \\
3.13\end{array}$ & 0.005 \\
\hline DGLA, 20:3 n-6 & $\begin{array}{l}2.89 \pm \\
0.92\end{array}$ & $\begin{array}{l}3.03 \pm \\
0.90\end{array}$ & 0.197 & $\begin{array}{l}2.84 \pm \\
1.06\end{array}$ & $\begin{array}{l}3.00 \pm \\
0.88\end{array}$ & 0.519 & $\begin{array}{l}2.90 \pm \\
0.98\end{array}$ & $\begin{array}{l}3.02 \pm \\
0.87\end{array}$ & 0.348 \\
\hline $\begin{array}{l}\text { Arachidonic acid, } \\
20: 4 \text { n-6 }\end{array}$ & $\begin{array}{l}12.03 \pm \\
1.78\end{array}$ & $\begin{array}{l}10.10 \pm \\
1.97\end{array}$ & $₫ 0.001$ & $\begin{array}{l}11.73 \pm \\
1.66\end{array}$ & $\begin{array}{l}10.73 \pm \\
2.17\end{array}$ & 0.122 & $\begin{array}{l}12.12 \pm \\
1.97\end{array}$ & $\begin{array}{l}10.16 \pm \\
1.84\end{array}$ & $\bowtie 0.001$ \\
\hline EPA, 20:5 n3 & $\begin{array}{l}0.29 \\
{[0.21} \\
0.41]\end{array}$ & $\begin{array}{l}0.33 \\
{[0.22} \\
0.47]\end{array}$ & 0.284 & $\begin{array}{l}0.34 \\
{[0.26} \\
0.43]\end{array}$ & $\begin{array}{l}0.29 \\
{[0.21} \\
0.43]\end{array}$ & 0.255 & $\begin{array}{l}0.29 \\
{[0.21} \\
0.40]\end{array}$ & $\begin{array}{l}0.34 \\
{[0.22} \\
0.46]\end{array}$ & 0.744 \\
\hline $\begin{array}{l}\text { Adrenic acid, } \\
22: 4 n-6\end{array}$ & $\begin{array}{l}0.46 \\
{[0.35} \\
0.53]\end{array}$ & $\begin{array}{l}0.41 \\
{[0.32} \\
0.49]\end{array}$ & 0.456 & $\begin{array}{l}0.40 \\
{[0.34} \\
0.48]\end{array}$ & $\begin{array}{l}0.42 \\
{[0.35} \\
0.52]\end{array}$ & 0.948 & $\begin{array}{l}0.46 \\
{[0.34} \\
0.56]\end{array}$ & $\begin{array}{l}0.41 \\
{[0.35} \\
0.47]\end{array}$ & 0.089 \\
\hline DPA, 22:5 n-3 & $\begin{array}{l}0.57 \\
{[0.46} \\
0.66]\end{array}$ & $\begin{array}{l}0.55 \\
{[0.45} \\
0.66]\end{array}$ & 0.817 & $\begin{array}{l}0.64 \\
{[0.48} \\
0.67]\end{array}$ & $\begin{array}{l}0.53 \\
{[0.45} \\
0.65]\end{array}$ & 0.172 & $\begin{array}{l}0.57 \\
{[0.45} \\
0.67]\end{array}$ & $\begin{array}{l}0.55 \\
{[0.47} \\
0.65]\end{array}$ & 0.091 \\
\hline DHA, 22:6 n-3 & $\begin{array}{l}2.88 \pm \\
0.83\end{array}$ & $\begin{array}{l}2.82 \pm \\
0.91\end{array}$ & 0.506 & $\begin{array}{l}2.94 \pm \\
0.81\end{array}$ & $\begin{array}{l}2.83 \pm \\
0.89\end{array}$ & 0.855 & $\begin{array}{l}2.91 \pm \\
0.78\end{array}$ & $\begin{array}{l}2.81 \pm \\
0.94\end{array}$ & 0.447 \\
\hline \multicolumn{10}{|c|}{$\begin{array}{l}\text { Data are presented as mean } \pm \text { SD or median [interquartile range], depending on the distribution. } \mathrm{P} \text { values denote probability } \\
\text { trends upon general linear model of difference in parameter distribution between major homozygote and minor allele carriers. } \\
\text { Models are adjusted for age, gender, PUFA/SFA [g] and BMl. Bolded text denotes statistical significance. }\end{array}$} \\
\hline
\end{tabular}

The rs174576 minor allele carriers were with significantly higher LA content than major allele homozygotes $(p=0.005)$, and rs174616 minor A allele carriers tended to be with higher LA levels $(p=0.063)$.

The AA content in plasma phospholipids was significantly lower in minor allele carriers for rs 174593 and rs 174576 (pख0.001 for both), while for rs174616 difference did not reach significance.

Other individual fatty acids were homogenously distributed across FADS2 genotypes in dominant genetic model.

The activity of rate-limiting desaturase-5 enzyme was lower in rs174593 and rs174576 minor allele carriers ( $p \otimes 0.001$ and $p=0.001$, respectively). The same subjects, presented with lower AA/EPA ratio in plasma phospholipids $(p=0.007$ and 0.034 , respectively) (Table 4). 
Table 4

Distribution of fatty acid metabolic conversion factors according to FADS2 genotypes in Serbian adults

\begin{tabular}{|c|c|c|c|c|c|c|c|c|c|}
\hline & \multicolumn{3}{|l|}{ rs174593 } & \multicolumn{3}{|l|}{ rs174616 } & \multicolumn{3}{|l|}{ rs174576 } \\
\hline & $\begin{array}{l}\text { TT } \\
\text { n } 37\end{array}$ & $\begin{array}{l}\mathrm{TC}+\mathrm{CC} \\
\mathrm{n} 51\end{array}$ & $\begin{array}{l}\mathrm{p}- \\
\text { value }\end{array}$ & $\begin{array}{l}\text { GG } \\
\text { n } 18\end{array}$ & $\begin{array}{l}\mathrm{GA}+\mathrm{AA} \\
\mathrm{n} 70\end{array}$ & $\begin{array}{l}\mathrm{p} \text { - } \\
\text { value }\end{array}$ & $\begin{array}{l}\mathrm{CC} \\
\text { n } 33\end{array}$ & $\begin{array}{l}\mathrm{CA}+\mathrm{AA} \\
\mathrm{n} 55\end{array}$ & $\begin{array}{l}\mathrm{p} \text { - } \\
\text { value }\end{array}$ \\
\hline D6D & $\begin{array}{l}0.12[0.09, \\
0.17]\end{array}$ & $\begin{array}{l}0.13[0.10 \\
0.15]\end{array}$ & 0.595 & $\begin{array}{l}0.11[0.09, \\
0.17]\end{array}$ & $\begin{array}{l}0.12[0.09 \\
0.15]\end{array}$ & 0.960 & $\begin{array}{l}0.12[0.09 \\
0.17]\end{array}$ & $\begin{array}{l}0.12[0.10, \\
0.15]\end{array}$ & 0.576 \\
\hline D5D & $\begin{array}{l}4.19[3.13 \\
5.70]\end{array}$ & $\begin{array}{l}3.32[2.78, \\
4.27]\end{array}$ & $\otimes 0.001$ & $\begin{array}{l}4.83[3.02, \\
5.89]\end{array}$ & $\begin{array}{l}3.67[2.88, \\
4.81]\end{array}$ & 0.108 & $\begin{array}{l}4.80[3.13 \\
5.70]\end{array}$ & $\begin{array}{l}3.37[2.79, \\
3.97]\end{array}$ & 0.001 \\
\hline AA/EPA & $\begin{array}{l}35.42 \\
{[29.47} \\
57.43]\end{array}$ & $\begin{array}{l}29.30 \\
{[20.27} \\
44.16]\end{array}$ & 0.007 & $\begin{array}{l}33.44 \\
{[28.76,} \\
35.42]\end{array}$ & $\begin{array}{l}35.75 \\
{[23.53} \\
55.31]\end{array}$ & 0.735 & $\begin{array}{l}35.26 \\
{[29.85,} \\
59.39]\end{array}$ & $\begin{array}{l}29.18 \\
{[20.58} \\
44.48]\end{array}$ & 0.034 \\
\hline $\mathrm{AA} / \mathrm{DHA}$ & $\begin{array}{l}4.46[3.57, \\
4.97]\end{array}$ & $\begin{array}{l}3.52[3.06 \\
4.63]\end{array}$ & 0.123 & $\begin{array}{l}3.99[3.56, \\
4.69]\end{array}$ & $\begin{array}{l}3.91[3.18 \\
4.93]\end{array}$ & 0.678 & $\begin{array}{l}4.16[3.56 \\
4.93]\end{array}$ & $\begin{array}{l}3.61[2.97, \\
4.89]\end{array}$ & 0.167 \\
\hline \multicolumn{10}{|c|}{$\begin{array}{l}\text { Data are as median [interquartile range]. } P \text { values denote probability trends upon general linear model of difference in } \\
\text { parameter distribution between major homozygote and minor allele carriers. Models are adjusted for age, gender, PUFA/SFA [g] }\end{array}$} \\
\hline
\end{tabular}

\section{Distribution of cardiometabolic parameters across FADS2 genotypes}

With regards to minor allele presence, there were no variations in distributions of cardio-metabolic risk factors within the subjects, inclusive of serum lipids and anthropometric parameters (Supplementary Table 3).

\section{Association between FADS2 genotypes and fatty acid status in plasma phospholipids}

In further regression models we analyzed individual plasma fatty acids and conversion factors that showed significant distribution variability in a dominant genetic model (Table 3, 4, Supplementary Table 2, 3), as following: palmitoleic acid, LA, AA, desaturase-5 and AA/EPA.

Upon multivariable-adjusted sequential regression models, minor allele addition of rs 174616 tended to be $(p=0.077)$ and rs174576 was associated $(p=0.003)$ with 0.99 and 1.64 higher LA content, respectively (Table 5, Fig. 1). Addition of minor allele was associated with lower AA and desaturase-5 levels, for each individual FADS2 variant examined, remaining significant upon Bonferroni correction for multiple testing (Table 5). The associations were independent of gender, age, ratio of polyunsaturated to saturated fat intake and obesity. Irrespective of demographics, fat intake and obesity, addition of rs174593 and rs174576 minor allele was associated with lower AA/EPA ratio, however not withstanding multiple testing $(p=0.022$ and $p=0.026$, respectively) (Table 5, Fig. 1). 
Table 5

Associations between FADS2 variants and plasma phospholipid fatty acids and conversion factors in Serbian adults

\begin{tabular}{|c|c|c|c|c|c|c|c|c|c|c|}
\hline & \multicolumn{2}{|c|}{$\begin{array}{l}\text { Palmitoleic acid, } \\
\text { 16:1 n-7 }\end{array}$} & \multicolumn{2}{|c|}{$\begin{array}{l}\text { Linoleic acid, 18:2 } \\
n-6\end{array}$} & \multicolumn{2}{|c|}{$\begin{array}{l}\text { Arachidonic acid, } \\
20: 4 \text { n-6 }\end{array}$} & \multicolumn{2}{|c|}{ Desaturase-5 } & \multicolumn{2}{|l|}{ AA/EPA } \\
\hline & $\beta(95 \% \mathrm{Cl})$ & $\begin{array}{l}\mathrm{p}- \\
\text { value }\end{array}$ & $\beta(95 \% \mathrm{Cl})$ & $\begin{array}{l}\mathrm{p}- \\
\text { value }\end{array}$ & $\beta(95 \% \mathrm{Cl})$ & $\begin{array}{l}\mathrm{p}- \\
\text { value }\end{array}$ & $\beta(95 \% \mathrm{Cl})$ & $\begin{array}{l}\mathrm{p} \text { - } \\
\text { value }\end{array}$ & $\begin{array}{l}\beta \\
(95 \% \mathrm{Cl})\end{array}$ & $\begin{array}{l}\mathrm{p}- \\
\text { value }\end{array}$ \\
\hline $\mathrm{M} 1: \Delta \mathrm{R}^{2}$ & 0.015 & & 0.024 & & 0.242 & & 0.176 & & 0.067 & \\
\hline rs174593 & $\begin{array}{l}-0.04 \\
(-0.12 \\
0.03)\end{array}$ & 0.278 & $\begin{array}{l}0.81 \\
(-0.33 \\
1.96)\end{array}$ & 0.161 & $\begin{array}{l}-1.67 \\
(-2.32 \\
-1.01)\end{array}$ & $\varangle 0.001$ & $\begin{array}{l}-0.11 \\
(-0.16 \\
-0.06)\end{array}$ & $\nabla 0.001$ & $\begin{array}{l}-0.10 \\
(-0.19 \\
-0.02)\end{array}$ & 0.019 \\
\hline $\mathrm{M} 2: \Delta \mathrm{R}^{2}$ & 0.089 & & 0.071 & & 0.025 & & 0.153 & & 0.036 & \\
\hline rs174593 & $\begin{array}{l}-0.03 \\
(-0.10 \\
0.05)\end{array}$ & 0.467 & $\begin{array}{l}0.78 \\
(-0.37 \\
1.93)\end{array}$ & 0.179 & $\begin{array}{l}-1.63 \\
(-2.30 \\
-0.96)\end{array}$ & $\otimes 0.001$ & $\begin{array}{l}-0.12 \\
(-0.17 \\
-0.07)\end{array}$ & $\otimes 0.001$ & $\begin{array}{l}-0.10 \\
(-0.19 \\
-0.02)\end{array}$ & 0.022 \\
\hline Age & $\begin{array}{l}0.01(0.00 \\
0.02)\end{array}$ & 0.031 & $\begin{array}{l}-0.05 \\
(-0.16 \\
0.06)\end{array}$ & 0.369 & $\begin{array}{l}0.02 \\
(-0.05 \\
0.08)\end{array}$ & 0.590 & $\begin{array}{l}0.00 \\
(-0.01 \\
0.00)\end{array}$ & 0.302 & $\begin{array}{l}0.00 \\
(-0.01 \\
0.01)\end{array}$ & 0.957 \\
\hline Gender & $\begin{array}{l}0.00 \\
(-0.10 \\
0.10)\end{array}$ & 0.975 & $\begin{array}{l}-0.36 \\
(-1.86 \\
1.14)\end{array}$ & 0.632 & $\begin{array}{l}0.12 \\
(-0.76 \\
1.00)\end{array}$ & 0.785 & $\begin{array}{l}-0.06 \\
(-0.13 \\
0.00)\end{array}$ & 0.064 & $\begin{array}{l}-0.08 \\
(-0.19 \\
0.04)\end{array}$ & 0.171 \\
\hline PUFA/SFA & $\begin{array}{l}-0.04 \\
(-0.12 \\
0.04)\end{array}$ & 0.348 & $\begin{array}{l}-0.70 \\
(-1.97 \\
0.57)\end{array}$ & 0.278 & $\begin{array}{l}-0.14 \\
(-0.89 \\
0.60)\end{array}$ & 0.702 & $\begin{array}{l}0.07 \\
(0.01 \\
0.12)\end{array}$ & 0.017 & $\begin{array}{l}-0.04 \\
(-0.13 \\
0.06)\end{array}$ & 0.467 \\
\hline BMI & $\begin{array}{l}0.01(0.00 \\
0.02)\end{array}$ & 0.255 & $\begin{array}{l}-0.10 \\
(-0.25 \\
0.05)\end{array}$ & 0.199 & $\begin{array}{l}0.06 \\
(-0.03 \\
0.15)\end{array}$ & 0.164 & $\begin{array}{l}-0.01 \\
(-0.02 \\
0.00)\end{array}$ & 0.003 & $\begin{array}{l}0.00 \\
(-0.01 \\
0.01)\end{array}$ & 0.789 \\
\hline $\mathrm{M} 1: \Delta \mathrm{R}^{2}$ & 0.008 & & 0.059 & & 0.120 & & 0.090 & & 0.015 & \\
\hline rs174616 & $\begin{array}{l}-0.03 \\
(-0.10 \\
0.04)\end{array}$ & 0.439 & $\begin{array}{l}1.19 \\
2.25)\end{array}$ & 0.028 & $\begin{array}{l}-1.11 \\
(-1.78 \\
-0.44)\end{array}$ & $\otimes 0.001$ & $\begin{array}{l}-0.07 \\
(-0.13 \\
-0.02)\end{array}$ & 0.006 & $\begin{array}{l}-0.05 \\
(-0.13 \\
0.04)\end{array}$ & 0.268 \\
\hline $\mathrm{M} 2: \Delta \mathrm{R}^{2}$ & 0.091 & & 0.052 & & 0.030 & & 0.143 & & 0.039 & \\
\hline rs174616 & $\begin{array}{l}-0.01 \\
(-0.09 \\
0.06)\end{array}$ & 0.737 & $\begin{array}{l}0.99 \\
(-0.11 \\
2.08)\end{array}$ & 0.077 & $\begin{array}{l}-1.09 \\
(-1.79 \\
-0.39)\end{array}$ & 0.003 & $\begin{array}{l}-0.08 \\
(-0.13 \\
-0.03)\end{array}$ & 0.002 & $\begin{array}{l}-0.05 \\
(-0.13 \\
0.04)\end{array}$ & 0.262 \\
\hline Age & $\begin{array}{l}0.01(0.00 \\
0.02)\end{array}$ & 0.026 & $\begin{array}{l}-0.05 \\
(-0.16 \\
0.06)\end{array}$ & 0.357 & $\begin{array}{l}0.03 \\
(-0.04 \\
0.10)\end{array}$ & 0.403 & $\begin{array}{l}0.00 \\
(-0.01 \\
0.00)\end{array}$ & 0.513 & $\begin{array}{l}0.00 \\
(-0.01 \\
0.01)\end{array}$ & 0.869 \\
\hline Gender & $\begin{array}{l}0.00 \\
(-0.10 \\
0.10)\end{array}$ & 0.966 & $\begin{array}{l}-0.55 \\
(-2.04 \\
0.94)\end{array}$ & 0.463 & $\begin{array}{l}0.39 \\
(-0.56 \\
1.34)\end{array}$ & 0.417 & $\begin{array}{l}-0.04 \\
(-0.11 \\
0.03)\end{array}$ & 0.239 & $\begin{array}{l}-0.06 \\
(-0.18 \\
0.05)\end{array}$ & 0.274 \\
\hline
\end{tabular}

p-values associated with multivariable hierarchical regression analysis examining associations between FADS2 variants and selected fatty acids in the additive genetic model. Bolded text denotes statistical significance.

AA, arachidonic acid; EPA, eicosapentaenoic acid; PUFA/SFA, ratio between daily intake of polyunsaturated to saturated fat; M1, M2, Model 1, 2.

AA/EPA and Desaturase-5 were log transformed to comply with normality of residuals for linear regression. 


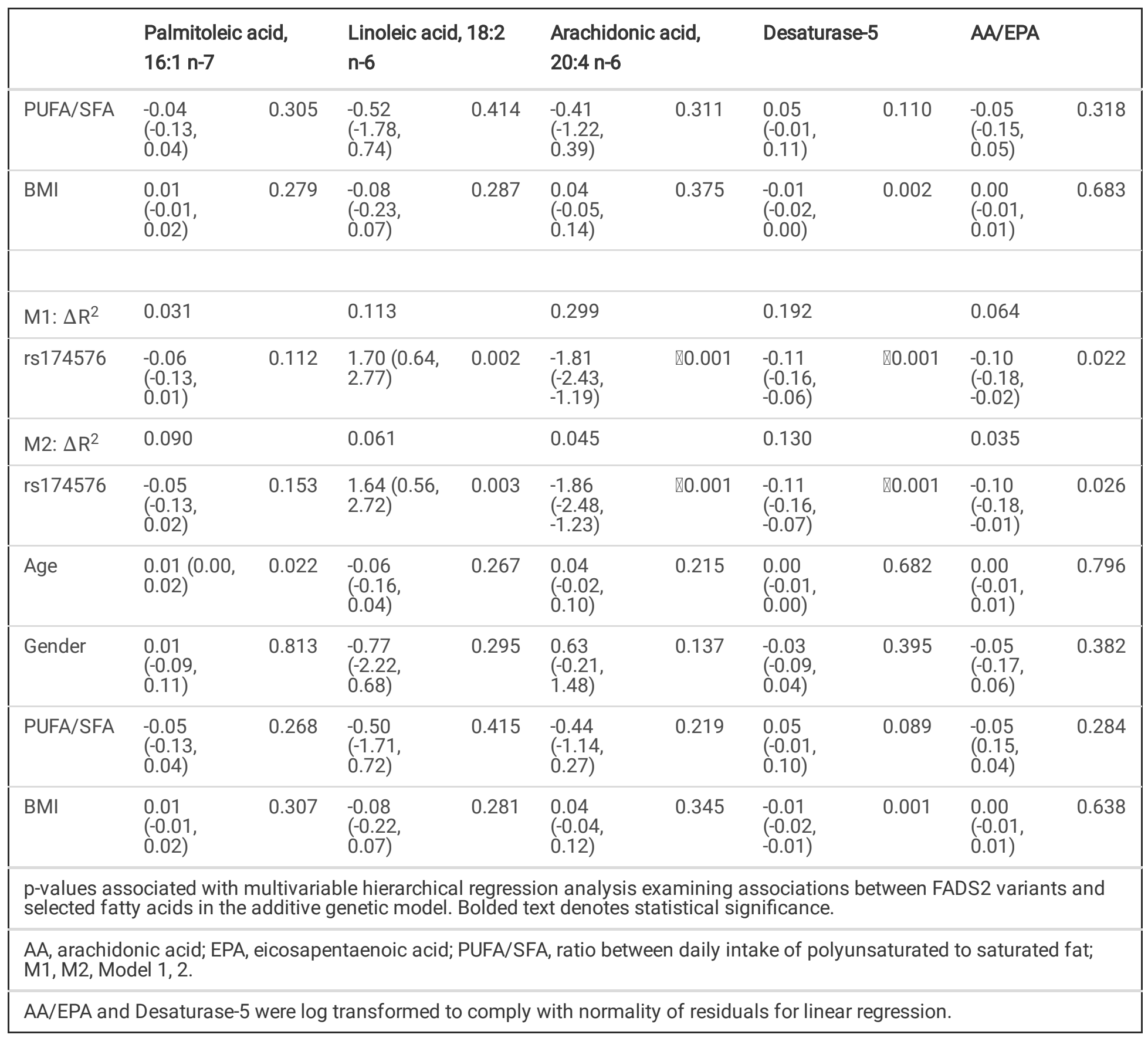

With regards to the included confounder, BMI showed an inverse association with the activity of desaturase-5, independent of the FADS2 variants.

\section{Sensitivity analysis: Implications for precision nutrition approach}

Multivariable regression models were additionally adjusted for individual FADS2 variants, to test whether the observed relationships of FADS2 variants were independent on each other. Only the rs 174576 remained associated with levels of $A A$ (multivariable-adjusted $\beta=-1.14$ (95\% Cl: $-2.25,-0.43), p=0.004$ ), in an additive genetic model (Fig. 2). The model explained $37 \%$ of plasma AA variability. The rs 174576 also remained associated with higher LA levels (multivariable-adjusted $\beta=1.98(95 \% \mathrm{Cl}$ : 0.40 , 3.56), $p=0.015)$, upon adjustment for the other two variants. No other associations survived, except for rs174593 remaining borderline related with estimated desaturase-5 activity $(p=0.045)$.

\section{Discussion}

Results from the current candidate-gene study in Serbian subjects comply with previous results from larger cohorts in other populations, showing link between minor allele presence within FADS genes and extensive metabolic conversion towards long- 
chain fatty acid products. In our study, FADS2 variants: rs174593, rs174616 and rs174576, were associated with LA, AA and estimated desaturase- 5 activity, as well as AA/EPA ratio in a sample of Serbian adults with no clinical sign of disease. Presence of the variants in FADS2 gene was associated with lower plasma levels of AA and desaturase-5 activity, but higher LA levels. The associations were independent of age, gender, ratio between intake of total polyunsaturated to saturated fats and obesity. We failed to demonstrate associations with n-3 very long-chain DHA, in line with previous studies that reported FADS1-2 gene associations with AA and found no associations with DHA $[11,13]$. The rs 174576 exhibited the strongest associations, putting into focus the variant's utilization in nutrigenetic-based precision nutrition strategies.

In our study rs 174593 , rs 174616 and rs 174576 variants explained $26.7 \%, 15 \%$ and $34.4 \%$ of AA variability in plasma phospholipids, respectively, which is in line with previously reported $28 \%$ of serum phospholipid AA variability explained by FADS1FADS2 cluster in a population of 727 Caucasian adults (20-64 years) [13]. Genome-wide association study from Framingham Heart Study's Offspring Cohort, examined the associations between 2.5M variants with RBC content of LC-PUFA (AA, EPA and DHA) in 2633 subjects [34]. This study showed AA levels to be significantly associated with as much as 82 variants, including FADS, which explained $13 \%$ of AA variability in RBC membranes [34]. Verona Heart Project reported associations between FADS1-3 genes and AA content in RBC and serum phospholipids in Italian middle-aged adults, while failed to demonstrate the associations with EPA and DHA [22]. In a recent paper, strong genetic background of AA variability, and to some extent weak link with EPA and DHA ratio is outlined as recurring theme [35]. Although we observed no FADS2 genotype-dependent differences in plasma EPA and DHA, the multivariable adjusted regression showed lower AA/EPA levels with the addition of rs174593 and rs174576 rare alleles, in line with metabolic competition between n-3 and n-6 anabolism. Similar to our findings, FADS candidate-gene study in healthy Caucasian adults, reported AA serum phospholipid content to be strongly genetically predetermined, but also failed to demonstrate associations with DHA [13]. Yet in another study, DHA/EPA conversion factor was associated with rs174576 [18] in females of Caucasian descent. Unlike quantitative measurements [18], we reported semi-quantitative plasma ratios of individual fatty acids expressed as a percentage of the total pool, potentially blurring the association with very LC-PUFA inclusive of DHA. However, metabolic conversion from dietary ALA towards ultimate n-3 products and DHA is known to be substantially low [36], and DHA tissue levels result from nutritional intake [13], supporting initiatives towards intensive n-3 intake, independently on genetic background.

Our findings comply with results from larger cohorts enrolling diverse ethnicities. The rs 174593 variant carriers presented with lower plasma AA and higher LA levels in 878 subjects from Europe and Far East [37]. Concordant with our results, in 1144 European adolescents, rs174616 minor allele presence was associated with higher LA and lower AA serum levels and lower desaturase-5 activity [38]. In the same study none of the 11 examined FADS variants was associated with DHA [38], compliant with our results. In Caucasian young adults residing in Canada, rs174576 minor allele carriers presented with significantly lower AA/LA levels in plasma, and lower desaturase-5 activity [17], similar to our findings. Contrary to reported previously [11, 14] we observed no associations with EPA levels. Lack of associations with long-chain n-3 metabolic products in our study, might result from baseline low n-3 intake. Namely, our subjects consumed a daily median of $190 \mathrm{mg}$ of total marine fatty acids below the recommended combined EPA + DHA intake of $500 \mathrm{mg}$ for cardiac health and other health benefits, according to the 2004 International Society for the Study of Fatty Acids and Lipids expert opinion. Previously, FADS1 rs174537 minor allele carriers were with lower desaturase- 5 activities at baseline, which however increased with increasing fish oil supplementation dose upon 6 months [16]. The finding indicates important nutrigenetic implication of protective FADS variants, penetrating with enhanced n-3 intake, ultimately supporting n-3 policies.

We found no differences in distributions of cardiometabolic risk factors with regards to minor alleles' presence in FADS2 gene. Our study group included non-diabetic subjects with no signs of clinical disease. In the complete sample, there were $15.9 \%, 18.2 \%$ and $17 \%$ of study subjects with elevated fasting glucose, triglycerides and total cholesterol respectively, while $13.6 \%$ of the subjects had decreased HDL-c. Narrow ranges for cardiometabolic risk factors, might to some extent underpin the absence of the associations, and future research is warranted in larger groups of dyslipidemic subject and those suffering from metabolic syndrome. Similar to our results, there were no significant associations among three selected FADS1-FADS2 variants [rs174537, rs174561 and rs3834458] and total cholesterol, LDL-c, HDL-c and triglycerides [16], although minor allele carriers were presented with lower plasma LC-PUFA levels and desaturase activities [16], examined in healthy subjects of mixed origin. Another study examined the relationship between 8 FADS variants (in FADS1, FADS2 and FADS3 genes), total cholesterol and triglycerides 
among 500 control subjects free of coronary artery disease [39]. Although no associations were found for triglyceride distribution, FADS2 rs 174575 minor allele homozygotes were with significantly lower total cholesterol levels in comparison with major homozygotes [39]. The latter study was conducted in Chinese Han population, and the ethnic-specific context is of detrimental role in studying FADS associations $[8,17]$. Notably, previous genome-wide association studies confirm relationship between FADS cluster and total cholesterol, HDL-c and LDL-c [40, 41]. Hence lack of the associations in our study must be considered with caution as serum lipids represent complex polygenic traits and our study might have lacked sufficient power to detect the link.

We found no differences in cumulative marine fatty acid, n-3, n-6 intake and respective ratio, between major homozygotes and minor allele carriers for FADS2 variants. We also observed no differences in intake distribution for individual fatty acids, with regards to minor allele presence across the three SNPs. Balanced habitual intake across FADS2 genotypes adds value to the independence of the FADS2 associations observed in our study.

Our findings challenge precision nutrition perspectives. In our study mean AA plasma levels were $10.91 \pm 2.11$. The addition of minor allele for rs174593, rs174616 and rs174576 resulted in $-1.63,-1.09$ and -1.81 lower AA levels, indicating physiologically relevant associations between FADS2 variants and circulating AA levels. The AA is with pro-inflammatory power, but its ultimate role appears to be dependent on eicosanoid balance [1]. Thus, the FADS2 variants associated with AA levels might modulate inflammation-triggered disease, as also indicated previously [11]. In our study rs174576 exhibited the strongest association, and its validation in larger cohorts is warranted for use in precision nutrition practice when prescribing balanced PUFA consumption (Fig. 2). The genotype aids in assigning individuals carrying minor allele as lower and major allele homozygotes as higher converters. Aside from genotype, personalized dietary practice must account for other lifestyle factors, starting with habitual n-6/n3 dietary ratio and plasma levels of LC-PUFA (Fig. 2). Controversy remains whether n-3 dietary supplementation would be of prominent power to overcome potential proinflammatory properties of AA-derived prostaglandins among null allele carriers [42].

Population subgroups might benefit from precision nutrition strategies. In our study, FADS2 variants and BMI were inversely associated with desaturase-5, indicating the potential of genetically-determined enhanced fatty acid conversion in weight management strategies. Within the Mediterranean population at high cardiovascular risk, desaturase-5 is inversely and desaturase- 6 activity adversely associated with the risk of metabolic syndrome [43], and confirmatory FADS2 gene studies in subjects who are overweight are needed. Lower AA levels and desaturase- 6 activity were previously reported in obese children carrying Thr54 variant in FABP2 gene [44], the latter known to be expressed in enterocytes regulating fatty acid absorption. The relevance of FADS2 gene in dramatically increasing pediatric obesity is to be confirmed. EPIC-Potsdam Study cohort study reports that estimated desaturase- 5 and desaturase- 6 activities are associated in the opposite direction with insulin resistance and diabetic risk in European populations [45]. The same was confirmed among middle-aged men participating Kuopio Ischemic Heart Disease Risk Factor Study [46], indicating that diabetic subjects carrying at least one variant allele might benefit more from n-3 dietary interventions. Further on, non-alcoholic steatohepatitis affects liver desaturase enzyme activities [47], an example of the reverse influence of disease towards endogenous fatty acid distribution. Results from Uppsala Longitudinal Study of Adult Men report that changes in fatty acid composition and desaturase activities occur long before the onset of metabolic syndrome [48]. Taken together, the results indicate the importance of intermediary phenotype management for primary prevention and support the use of genetically-determined fatty acid levels as predictive biomarkers in chronic disease management. A recent review paper indicates perspectives in precision nutrition based on tailoring PUFA supply according to genetic structure, among individuals or subpopulations [12].

Our study results support FADS2 rs174576 gene variation as a potential nutrigenetic hallmark to be used in the precision nutrition concept. In practical terms, subjects who are homozygotes for rs174576 major allele linked with higher endogenous AA levels, might benefit more from n-3 based dietary interventions, especially if their habitual intake inclines towards higher n-6/n-3 ratio (Fig. 2). Previous studies encourage provision of personalized genetic advice for improvement of dietary habits with relation to omega-3 intake $[49,50]$. Acknowledgment of genetic information was associated with raised awareness on health-promoting power of n-3 intake [49]. Due to recognized health benefits of EPA and DHA intake [51, 52], yet its low intake in developed and developing countries [53], alternative measures of motivating people to increase n-3 intake are highly desirable [49] and nutrigenetic FADS information might have a leveraging effect in personalized dietary modifications.

Page $15 / 22$ 


\section{Strengths and limitations}

Limited sample size constraints us in commenting on the generalizability of our results in Serbians, and larger gene-associations studies are warranted in the population. We acknowledge the inherent limitations of $24 \mathrm{~h}$ recall, as retrospective dietary assessment method based on the subjective report. In order to improve report accuracy several strategies were employed: intensive training of the interviewers, non-hurried pace of interview, application of pre-tested probing questions and multiple-pass approach. The cause-effect relationship could not be claimed in our study, nor may residual confounding be ruled out. We however included homogenous sample, with all subjects apparently healthy, of Serbian ethnicity and residing in urban area. In the regression models we controlled for age, gender and intake of fat, thus minimizing possible effect modification due to uncontrolled factors. Due to the $70 \%$ subjects who are overweight in our sample, we also controlled for obesity.

\section{Conclusion}

To the best of our knowledge, we are the first to report associations between FADS variants and fatty acid metabolism in Serbians. In our study, FADS2 rs174593, rs174616 and rs174576 variants were associated with higher linoleic, but lower arachidonic acid, arachidonic acid to eicosapentaenoic acid ratio and estimated desaturase- 5 activity in plasma phospholipids. Subjects carrying FADS2 null alleles were with higher conversion capacity towards long-chain products and might benefit more from omega-3 dietary interventions. Given low fish intake in our country, affirmative strategies towards omega-3 enforcement are highly desirable. In light with the previous, our study indicates rs174576 in FADS2 gene as a potential nutrigenetic biomarker for precision nutrition actions aimed at PUFA supply. Future larger cohorts and intervention studies with varying omega-6/omega-3 intake ratio should validate the biomarker and examine its associations with disease.

\section{Declarations}

Ethics approval and consent to participate

Current study formed part of a larger interventional study registered at ClinicalTrials.gov as NCT02800967. The study protocol adhered to the regulations of the 1975 Declaration of Helsinki and is approved by the Clinical Hospital Center in Zemun (Belgrade, Serbia), Ethics Committee Approval, No: 2125, 2013.

\section{Consent for publication}

Not applicable.

\section{Availability of data and materials}

The datasets generated and/or analysed during the current study are not publicly available due to the confidentiality of data and the assurance provided to the participants, in addition to the protection of potential harm (including psychological harm, social harm, and stigmatization), but are available from the corresponding author on reasonable request

\section{Competing interests}

The authors declare that they have no competing interests. 
The authors acknowledge financial support from the Ministry of Education and Science of Serbia, project no. 41030 and 41028 and EU FP7 project BACCHUS (Grant agreement $\left.n^{\circ} 312090\right)$.

\section{Authors' contributions}

MMZ, conceived and performed the study, collected DNA samples from the participants, analyzed and interpreted results, assisted with gas chromatography and drafted the manuscript. LjS, conceptualized genetic work and genetic examinations. MZ, performed collection, compilation and analysis of dietary data. BP, carried out genetic analysis and analysis of fatty acids in plasma. LjS, MZ, $\mathrm{BP}$ and MŽ critically revised manuscript. MŽ, AS and MG, acquired financial resources and conceptualized the work. All authors read and approved the final manuscript.

\section{Acknowledgements}

The Authors are grateful to study participants for taking part in the study, and study personnel involved during the course of the study.

\section{References}

1.

Sonnweber T, Pizzini A, Nairz M, Weiss G, Tancevski I. Arachidonic Acid Metabolites in Cardiovascular and Metabolic Diseases. Int J Mol Sci. 2018;19:3285.

2.

Serhan CN. Novel pro-resolving lipid mediators in inflammation are leads for resolution physiology. Nature. 2014;510:92-101.

3.

Marszalek JR, Lodish HF. Docosahexaenoic acid, fatty acid interacting proteins, and neuronal function: breastmilk and fish are good for you. Annu Rev Cell Dev Biol. 2005;21:633-57.

4.

Vessby B. Dietary fat, fatty acid composition in plasma and the metabolic syndrome. Curr Opin Lipidol. 2003;14:15-9.

5 .

Jackson KH, Harris WS. Blood Fatty Acid Profiles: New Biomarkers for Cardiometabolic Disease Risk. Curr Atheroscler Rep. 2018;20:22.

6.

Zec MM, Schutte AE, Ricci C, Baumgartner J, Kruger IM, Smuts CM. Long-Chain Polyunsaturated Fatty Acids Are Associated with Blood Pressure and Hypertension over 10-Years in Black South African Adults Undergoing Nutritional Transition. Foods.

2019;8:394.

7.

Kompauer I, Demmelmair H, Koletzko B, Bolte G, Linseisen J, Heinrich J, et al. Association of fatty acids in serum phospholipids with hay fever, specific and total immunoglobulin E. Br J Nutr. 2005;93:529-35.

8.

Harris DN, Ruczinski I, Yanek LR, Becker LC, Becker DM, Guio H, et al. Evolution of hominin polyunsaturated fatty acid metabolism: from africa to the new world. Genome Biol Evol. 2019;11:1417-30.

9.

Lee JM, Lee H, Kang S, Park WJ. Fatty acid desaturases, polyunsaturated fatty acid regulation, and biotechnological advances. Nutrients. 2016;8:23.

10.

Lankinen M, Uusitupa M, Schwab U. Genes and Dietary Fatty Acids in Regulation of Fatty Acid Composition of Plasma and Erythrocyte Membranes. Nutrients. 2018;10:1785.

11.

Page 17/22 
O'Neill CM, Minihane AM. The impact of fatty acid desaturase genotype on fatty acid status and cardiovascular health in adults. Proc Nutr Soc. 2017;76:64-75.

12.

Koletzko B, Reischl E, Tanjung C, Gonzalez-Casanova I, Ramakrishnan U, Meldrum S, et al. FADS1 and FADS2 polymorphisms modulate fatty acid metabolism and dietary impact on health. Annu Rev Nutr. 2019;39:21-44.

13.

Schaeffer L, Gohlke H, Müller M, Heid IM, Palmer LJ, Kompauer I, et al. Common genetic variants of the FADS1 FADS2 gene cluster and their reconstructed haplotypes are associated with the fatty acid composition in phospholipids. Hum Mol Genets.

2006;15:1745-56.

14.

Lemaitre RN, Tanaka T, Tang W, Manichaikul A, Foy M, Kabagambe EK, et al. Genetic loci associated with plasma phospholipid n-3 fatty acids: a meta-analysis of genome-wide association studies from the CHARGE Consortium. PLoS Genet. 2011;7:e1002193. 15.

Tanaka T, Shen J, Abecasis GR, Kisialiou A, Ordovas JM, Guralnik JM, et al. Genome-wide association study of plasma polyunsaturated fatty acids in the InCHIANTI Study. PLoS Genet. 2009;5:e1000338.

16.

Al-Hilal M, Alsaleh A, Maniou Z, Lewis FJ, Hall WL, Sanders TA, et al. Genetic variation at the FADS1-FADS2 gene locus influences delta-5 desaturase activity and LCPUFA proportions after fish oil supplement. J Lipid Res. 2013;54:542e551.

17.

Merino DM, Johnston H, Clarke S, Roke K, Nielsen D, Badawi A, et al. Polymorphisms in FADS1 and FADS2 alter desaturase activity in young Caucasian and Asian adults. Mol Genet Metab. 2011;103:171-8.

18.

Abdelmagid SA, Clarke SE, Roke K, Nielsen DE, Badawi A, El-Sohemy A, et al. Ethnicity, sex, FADS genetic variation, and hormonal contraceptive use influence delta-5-and delta-6-desaturase indices and plasma docosahexaenoic acid concentration in young Canadian adults: a cross-sectional study. Nutr Metab. 2015;12:14.

19.

Chilton FH, Murphy RC, Wilson BA, Sergeant S, Ainsworth H, Seeds MC, et al. Diet-gene interactions and PUFA metabolism: a potential contributor to health disparities and human diseases. Nutrients. 2014;6:1993-2022.

20.

Simopoulos AP. An increase in the omega-6/omega-3 fatty acid ratio increases the risk for obesity. Nutrients. 2016;8:128.

21.

Calder PC. Long chain fatty acids and gene expression in inflammation and immunity. Curr Opin Clin Nutr Metab Care. 2013;16:425-33.

22.

Malerba G, Schaeffer L, Xumerle L, Klopp N, Trabetti E, Biscuola M, et al. SNPs of the FADS gene cluster are associated with polyunsaturated fatty acids in a cohort of patients with cardiovascular disease. Lipids. 2008;43:289-99.

23.

Mathias RA, Sergeant S, Ruczinski I, Torgerson DG, Hugenschmidt CE, Kubala M, et al. The impact of FADS genetic variants on omega6 polyunsaturated fatty acid metabolism in African Americans. BMC Genet. 2011;12:50.

24.

Sergeant S, Hugenschmidt CE, Rudock ME, Ziegler JT, Ivester P, Ainsworth HC, et al. Differences in arachidonic acid levels and fatty acid desaturase (FADS) gene variants in African Americans and European Americans with diabetes or the metabolic syndrome. $\mathrm{Br}$ J Nutr. 2012;107:547-55.

25.

Green RC, Berg JS, Grody WW, Kalia SS, Korf BR, Martin CL, et al. ACMG recommendations for reporting of incidental findings in clinical exome and genome sequencing. Genet Sci. 2013;15:565.

26.

Gurinović M, Milešević J, Kadvan A, Nikolić M, Zeković M, Djekić-lvanković M, et al. Development, features and application of DIET ASSESS \& PLAN (DAP) software in supporting public health nutrition research in Central Eastern European Countries (CEEC). Food 
Chem. 2018;238:186-94.

27.

Gurinović M, Milešević J, Kadvan A, Djekić-Ivanković M, Debeljak-Martačić J, Takić M, et al. Establishment and advances in the online Serbian food and recipe data base harmonized with EuroFIR standards. Food Chem. 2016;193:30-8.

28.

Knez M, Stangoulis JC, Zec M, Debeljak-Martacic J, Pavlovic Z, Gurinovic M, Glibetic M. An initial evaluation of newly proposed biomarker of zinc status in humans-linoleic acid: dihomo-y-linolenic acid (LA: DGLA) ratio. Clinical Nutr ESPEN. 2016;15:85-92.

29.

Folch J, Lees M, Sloane Stanley GH. A simple method for the isolation and purification of total lipides from animal tissues. J Biol Chem. 1957;226:497-509.

30.

Warensjo E, Rosell M, Hellenius ML, Vessby B, De Faire U, Riserus U. Associations between estimated fatty acid desaturase activities in serum lipids and adipose tissue in humans: Links to obesity and insulin resistance. Lipids Health Dis. 2009;8:37.

31.

Johnson AD, Handsaker RE, Pulit SL, Nizzari MM, O'Donnell CJ, De Bakker PI. SNAP: a web-based tool for identification and annotation of proxy SNPs using HapMap. Bioinformatics. 2008;24:2938-39.

32.

Boyle AP, Hong EL, Hariharan M, Cheng Y, Schaub MA, Kasowski M, et al. Annotation of functional variation in personal genomes using RegulomeDB. Genome Res. 2012;22:1790-97.

33.

Kunkel LM, Smith KD, Boyer SH, Borgaonkar DS, Wachtel SS, Miller OJ, et al. Analysis of human Y-chromosome-specific reiterated DNA in chromosome variants. Proc Natl Acad Sci U S A. 1997;74:1245-49.

34.

Tintle NL, Pottala JV, Lacey S, Ramachandran V, Westra J, Rogers A, et al. A genome-wide association study of saturated, monoand polyunsaturated red blood cell fatty acids in the Framingham Heart Offspring Study. Prostaglandins Leukot Essent Fatty Acids. 2015;94:65-72.

35.

WS

Harris

Nutrigenetics

2020

Harris WS. Nutrigenetics: Omega-3 and Omega-6 Fatty Acids. In: Principles of Nutrigenetics and Nutrigenomics. Academic Press; 2020. p. 223-226.

36.

Brenna JT, Salem N Jr, Sinclair A, Jr, Cunnane SC. Alpha-linolenic acid supplementation and conversion to n-3 long-chain polyunsaturated fatty acids in humans. Prostaglandins Leukot Essent Fat Acids. 2009;80:85-91.

37.

Roke K, Ralston JC, Abdelmagid S, Nielsen DE, Badawi A, El-Sohemy A, et al. Variation in the FADS1/2 gene cluster alters plasma n6 PUFA and is weakly associated with hsCRP levels in healthy young adults. Prostaglandins Leukot Essent Fatty Acids. 2013;89:257-63.

38.

Bokor S, Dumont J, Spinneker A, Gonzalez-Gross M, Nova E, Widhalm K, et al. Single nucleotide polymorphisms in the FADS gene cluster are associated with delta- 5 and delta- 6 desaturase activities estimated by serum fatty acid ratios. J Lipid Res. 2010;51:2325-33.

39.

Wu Y, Zeng L, Chen X, Xu Y, Ye L, Qin L, et al. Association of the FADS gene cluster with coronary artery disease and plasma lipid concentrations in the northern Chinese Han population. Prostaglandins Leukot Essent Fat Acids. 2017;117:11-6.

40. 
Kathiresan S, Willer CJ, Peloso GM, Demissie S, Musunuru K, Schadt EE, et al. Common variants at 30 loci contribute to polygenic dyslipidemia. Nat Genet. 2009;41:56-65.

41.

Sabatti C, Service SK, Hartikainen AL, Pouta A, Ripatti S, Brodsky J, et al. Genome-wide association analysis of metabolic traits in a birth cohort from a founder population. Nat Genet. 2009;41:35-46.

42.

Chilton FH, Dutta R, Reynolds LM, Sergeant S, Mathias RA, Seeds MC. Precision nutrition and omega-3 polyunsaturated fatty acids: A case for personalized supplementation approaches for the prevention and management of human diseases. Nutrients. 2017;9:1165.

43.

Mayneris-Perxachs J, Guerendiain M, Castellote Al, Estruch R, Covas MI, Fitó M, et al. Plasma fatty acid composition, estimated desaturase activities, and their relation with the metabolic syndrome in a population at high risk of cardiovascular disease. Clinical Nutr. 2014;33:90-7.

44.

Okada T, Sato NF, Kuromori Y, Miyashita M, Iwata F, Hara M, et al. Thr-Encoding allele homozygosity at codon 54 of fabp 2 gene may be associated with impaired delta 6 desatruase activity and reduced plasma arachidonic acid in obese children. J Atheroscler Thromb. 2006;13:192-6.

45.

Jacobs S, Schiller K, Jansen EH, Boeing H, Schulze MB, Kroger J. Evaluation of various biomarkers as potential mediators of the association between delta 5 desaturase, delta 6 desaturase, and stearoyl-coa desaturase activity and incident type 2 diabetes in the european prospective investigation into cancer and nutrition-potsdam study. Am J Clin Nutr. 2015;102:155-64.

46.

Yary T, Voutilainen S, Tuomainen TP, Ruusunen A, Nurmi T, Virtanen JK. Serum N-6 polyunsaturated fatty acids, delta5- and delta6desaturase activities, and risk of incident type 2 diabetes in men: The Kuopio ischaemic heart disease risk factor study. Am J Clin Nutr. 2016;103:1337-43.

47.

Vaittinen M, Mannisto V, Kakela P, Agren J, Tiainen M, Schwab U, et al. Interorgan cross talk between fatty acid metabolism, tissue inflammation, and fads2 genotype in humans with obesity. Obesity (Silver Spring). 2017;25:545-52.

48.

Warensjö E, Sundström J, Lind L, Vessby B. Factor analysis of fatty acids in serum lipids as a measure of dietary fat quality in relation to the metabolic syndrome in men. Am J Clin Nutr. 2006;84:442-8.

49.

Roke K, Walton K, Klingel S, Harnett A, Subedi S, Haines J, Mutch D. Evaluating changes in omega-3 fatty acid intake after receiving personal FADS1 genetic information: a randomized nutrigenetic intervention. Nutrients. 2017;9:240.

50.

Livingstone KM, Celis-Morales C, Navas-Carretero S, San-Cristobal R, Macready AL, Fallaize R, et al. Effect of an internet-based, personalized nutrition randomized trial on dietary changes associated with the Mediterranean diet: The Food4Me study. Am J Clin Nutr. 2016;104:288-97.

51.

Bell GA, Kantor ED, Lampe JW, Kristal AR, Heckbert SR, White E. Intake of long-chain n-3 fatty acids from diet and supplements in relation to mortality. Am J Epidemiol. 2014;179:710-20.

52.

Kim YS, Xun P, Iribarren C, Van Horn L, Steffen L, Daviglus ML, et al. Intake of fish and long-chain omega-3 polyunsaturated fatty acids and incidence of metabolic syndrome among American young adults: A 25-year follow-up study. Eur J Nutr. 2016;1-10. 53.

Forsyth S, Gautier S, Salem N Jr. Global estimates of dietary intake of docosahexaenoic acid and arachidonic acid in developing and developed countries. Ann Nutr Metab. 2016;68:258-67.

\section{Figures}

Page 20/22 

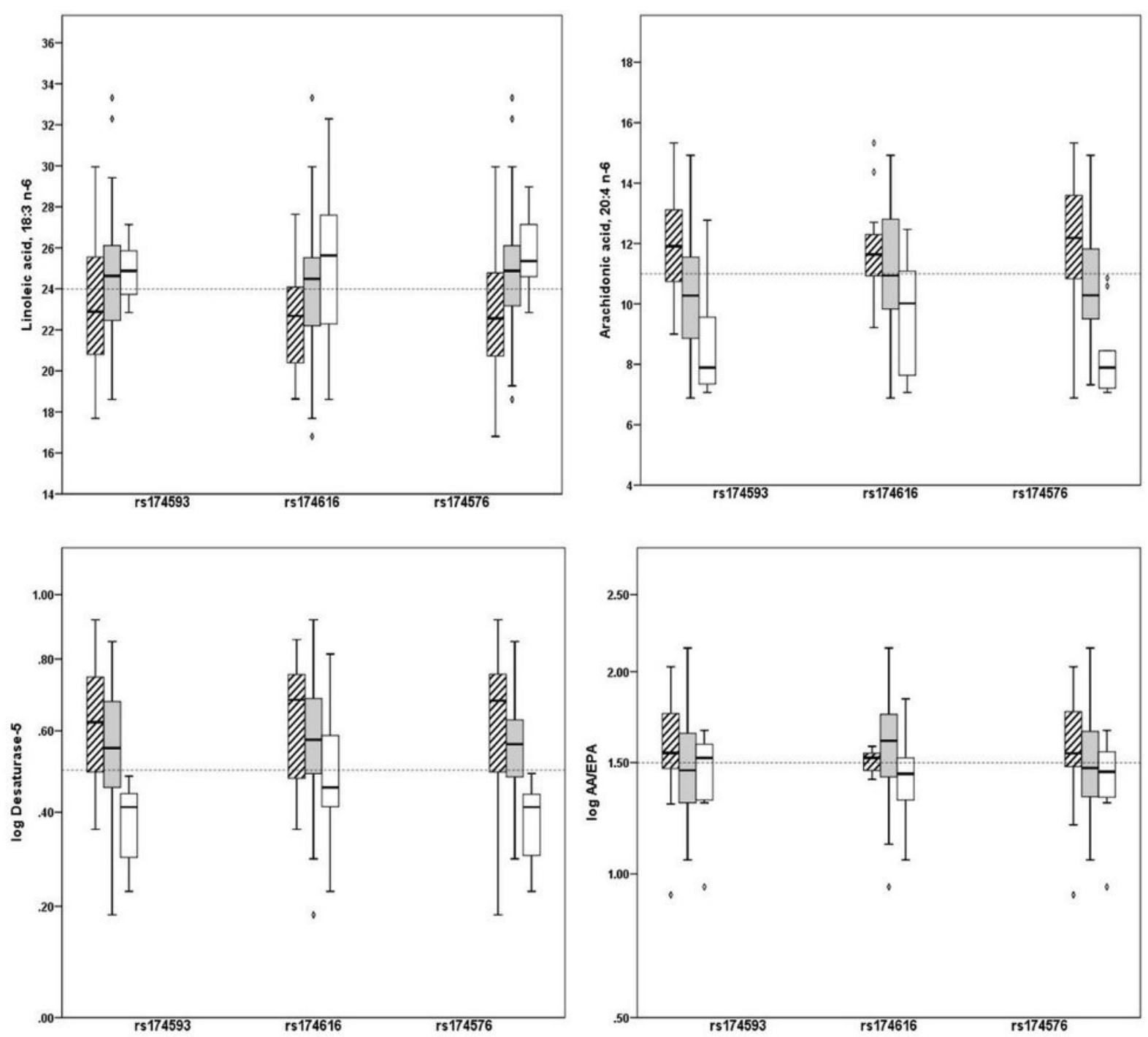

VIIII/, Major allele homozygote

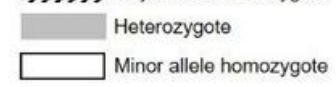

Figure 1

Associations between FADS2 variants and plasma phospholipid fatty acids and conversion factors in Serbian adults. 


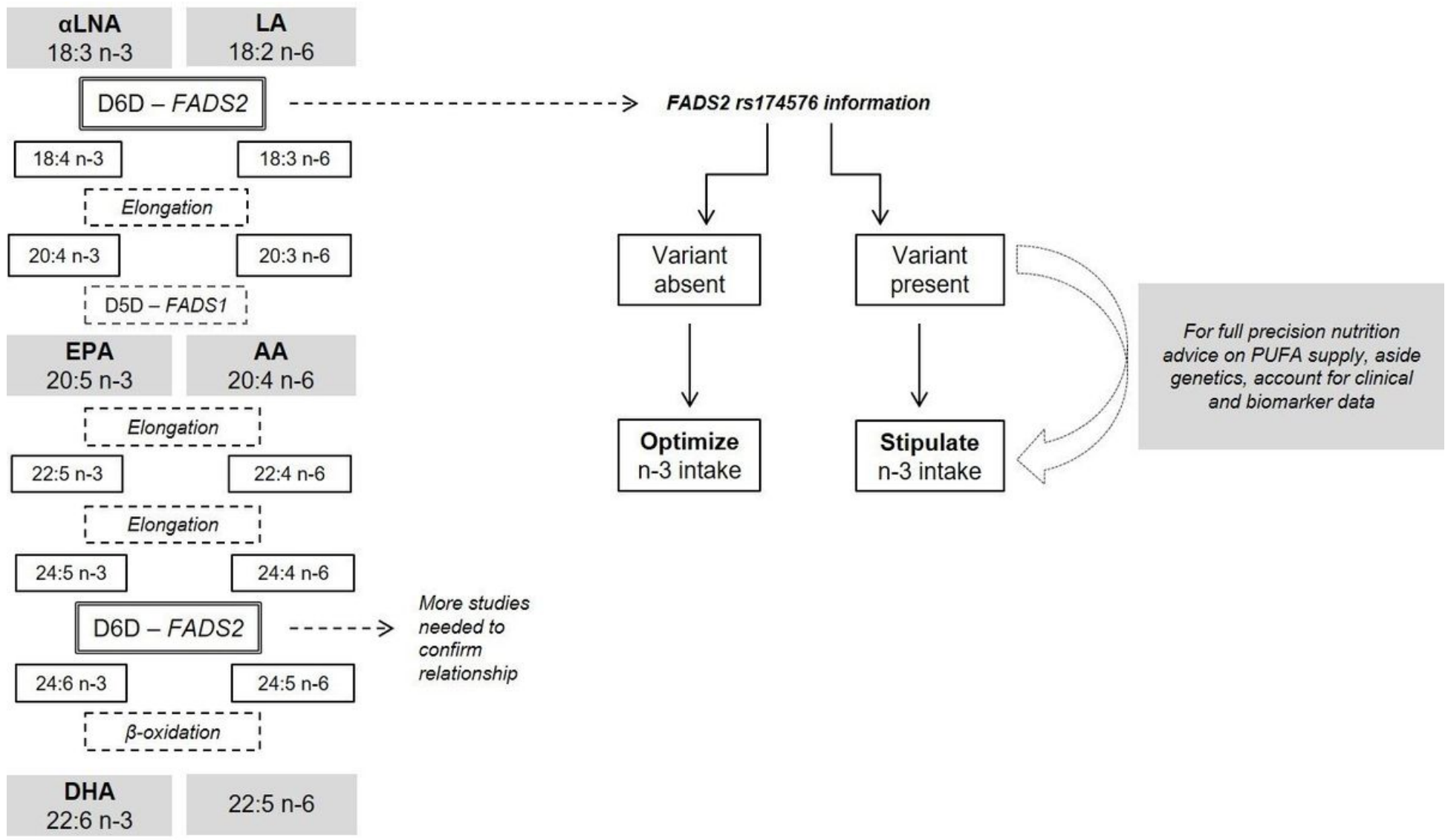

Figure 2

FADS2 rs174576 as candidate genetic variant for nutrigenetic-based precision nutrition practice for primary prevention

\section{Supplementary Files}

This is a list of supplementary files associated with this preprint. Click to download.

- SUPPLEMENTARYTABLES.docx 